\title{
Aortic Valve Predilatation with a Small Balloon, without Rapid Pacing, prior to Transfemoral Transcatheter Aortic Valve Replacement
}

\author{
Anupama Shivaraju, ${ }^{1,2}$ Christian Thilo, ${ }^{3}$ Neal Sawlani, ${ }^{2}$ Ilka Ott, ${ }^{1}$ Heribert Schunkert, ${ }^{1,4}$ \\ Wolfgang von Scheidt, ${ }^{3}$ Adnan Kastrati, ${ }^{1,4}$ and Albert Markus Kasel $\mathbb{C}^{1,3}$ \\ ${ }^{1}$ Deutsches Herzzentrum München, Department for Cardiovascular Diseases, Technische Universität München, Lazarettstr. 36, \\ 80636 Munich, Germany \\ ${ }^{2}$ Department of Cardiology, Advocate Christ Medical Center, 4440 W. 95th Street, Oak Lawn, IL, USA \\ ${ }^{3}$ Department of Cardiology, Klinikum Augsburg, Herzzentrum Augsburg-Schwaben, Stenglinstr. 2, 86156 Augsburg, Germany \\ ${ }^{4}$ Deutsches Zentrum für Herz- und Kreislaufforschung (DZHK), Munich Heart Alliance, Munich, Germany
}

Correspondence should be addressed to Albert Markus Kasel; markus_kasel@web.de

Received 16 July 2017; Revised 28 February 2018; Accepted 14 March 2018; Published 30 April 2018

Academic Editor: Giovanni Mariscalco

\begin{abstract}
Copyright (C) 2018 Anupama Shivaraju et al. This is an open access article distributed under the Creative Commons Attribution License, which permits unrestricted use, distribution, and reproduction in any medium, provided the original work is properly cited.
\end{abstract}

\begin{abstract}
Objectives. The aim of this study is to assess the feasibility and clinical outcome of transcatheter aortic valve replacement (TAVR) using aortic valve predilatation (AVPD) with a small, nonocclusive balloon. Background. Balloon aortic valvuloplasty (BAV) under rapid pacing is generally performed in TAVR to ensure the passage and sufficient deployment of the prosthesis in the stenotic AV. BAV may cause serious complications, such as left ventricular stunning or cerebrovascular embolism. Methods. A cohort of 50 consecutive patients with severe aortic stenosis underwent transfemoral TAVR with the Edwards Sapien 3-heart valve. All patients underwent AVPD with a small, nonocclusive balloon $(12 \times 60$ or $14 \times 60 \mathrm{~mm})$ without rapid pacing. Procedural data and clinical outcomes were analyzed. Results. The mean age of the cohort was $81 \pm 6$ years and the mean logistic EuroSCORE (European System for Cardiac Operative Risk Evaluation) was $13 \pm 9$. Crossing the AV and prosthesis implantation was successful in all cases. The postprocedural mean AV gradient was $12 \pm 5 \mathrm{mmHg}$. There were no cases of aortic regurgitation $\geq$ grade 2 . No periprocedural stroke occurred. One patient (2\%) with chronic atrial fibrillation displayed a transient Wernicke aphasia occurring more than 24 hours after TAVR. Mortality was $0 \%$ at 30 days after procedure. Conclusion. In TAVR, AVPD with a small, nonocclusive balloon can be safely performed. By avoiding rapid pacing, this technique may be a valid alternative to traditional BAV. Whether or not the use of APVD without rapid pacing translates into less periprocedural complications needs to be assessed in future studies.
\end{abstract}

\section{Introduction}

Transfemoral transcatheter aortic valve replacement (TAVR) has evolved into the standard of care for patients with severe, symptomatic aortic stenosis (AS) at intermediate, inoperable, or high surgical risk [1]. As device caliber and technological improvements continue to garner increasing attention, some cornerstones of transfemoral implantation techniques remain unchanged. Such principles are rapid pacing and balloon aortic valvuloplasty (BAV) prior to valve implantation $[2,3]$. However, the impact of transient ventricular stunning during rapid pacing is unclear, and BAV during TAVR may contribute to cerebral microembolization of calcified debris from the aortic valve $[4,5]$. The aim of our current study is to assess the safety and feasibility of AVPD prior to TAVR using a small and nonocclusive balloon without the use of rapid ventricular pacing.

\section{Methods}

2.1. Patient Selection and Preparation. From February 2014 until June 2014, we prospectively evaluated and treated 50 


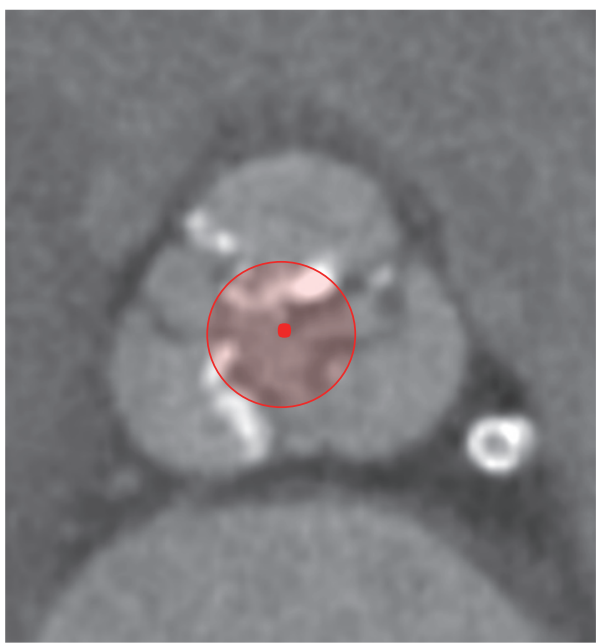

(a)

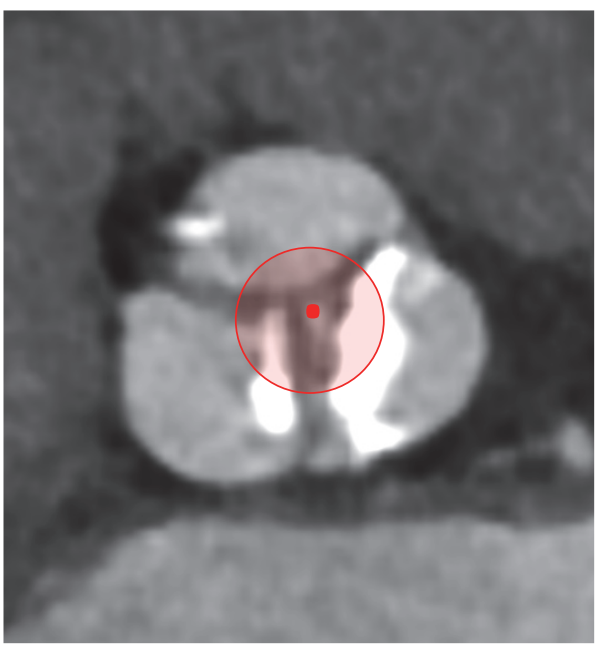

(c)

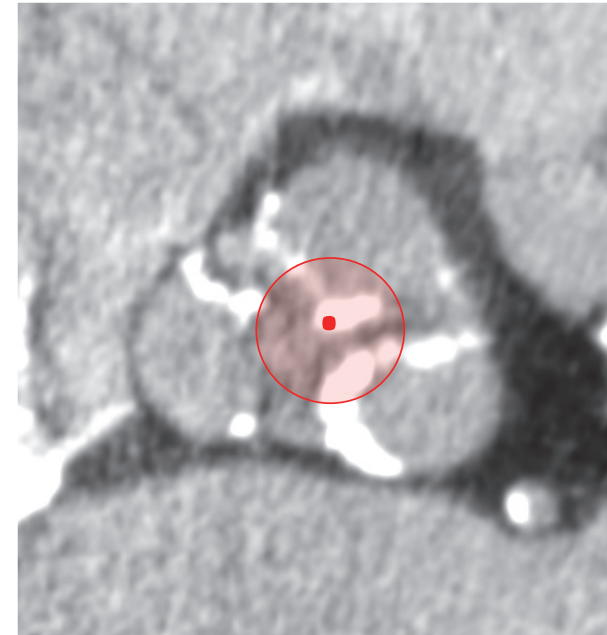

(b)

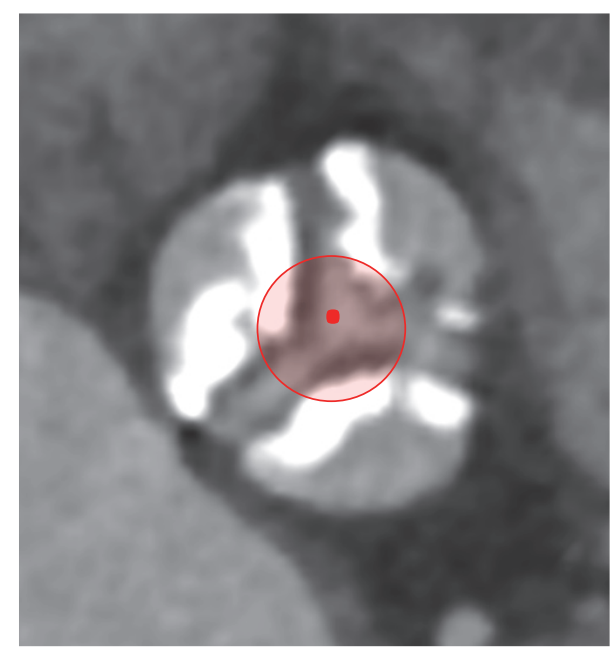

(d)

FIGURE 1: CT-imaging of aortic valves with different degree of calcification. Schematic effect of AVPD with a small, "nonocclusive" balloon.

consecutive patients with severe, symptomatic AS using AVPD prior to implantation of the Sapien 3 (Edwards Lifesciences, Irvine, CA, USA) transcatheter heart valve (THV).

All patients undergoing TAVR were evaluated with a transthoracic echo (TTE), coronary angiogram, and an ECG gated 384-slice, multidetector computed tomography angiogram (CTA) of the heart and thoracic, abdominal and bilateral lower extremity vasculature. At the time of the procedure, an aortic root and selective iliofemoral angiograms were performed. The procedure was performed under conscious sedation in majority of the cases. A TTE was obtained on all patients on postprocedure day one to assess the aortic valve anatomy and function including obtaining hemodynamic measurements.

2.2. TAVR Procedures. After crossing the AV, traditionally BAV is performed using an occlusive balloon $(20,23$, or $25 \times 40 \mathrm{~mm}$ ) under rapid ventricular pacing. In our study, however, a nonocclusive balloon $(12 \times 60$ or $14 \times 60 \mathrm{~mm}$;
Osypka AG, Rheinfelden-Herten, Germany) was used for AVPD (Figure 1). No rapid ventricular pacing was performed during AVPD. A comparison of balloon sizes in relation to aortic valve anatomy is provided in Figures 2(a) and 2(b). An example of pre- and post-AVPD hemodynamic data is shown in Figure 2(c). Thereafter, the Sapien 3 valve was inserted and positioned across the AV annulus under fluoroscopy guidance and deployed under rapid ventricular pacing.

2.3. Procedural Endpoints and Definitions. The primary endpoint was device success. The secondary endpoints were disabling stroke, nondisabling stroke, transient ischemic attack (TIA), and procedural mortality. Device success, disabling stroke, nondisabling stroke, TIA, and procedural mortality were all defined using the VARC2 definitions [6]. Device success was defined as absence of procedural mortality, correct positioning of a single prosthetic heart valve, and intended performance of the implanted prosthetic valve (no patient-prosthesis mismatch, mean AV gradient $<20 \mathrm{mmHg}$, 


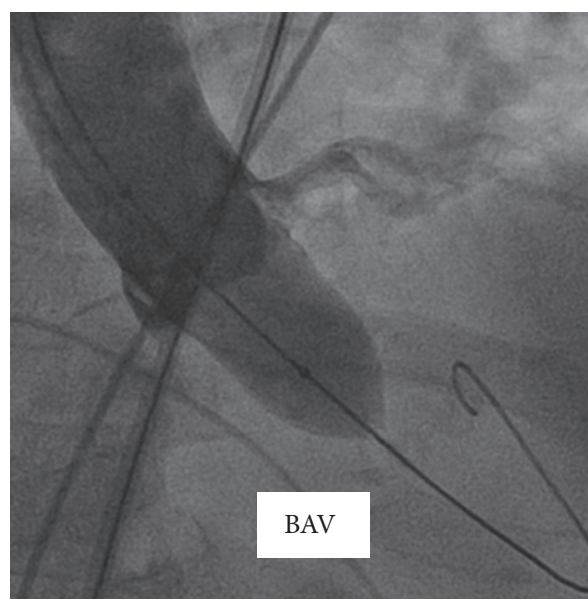

(a)

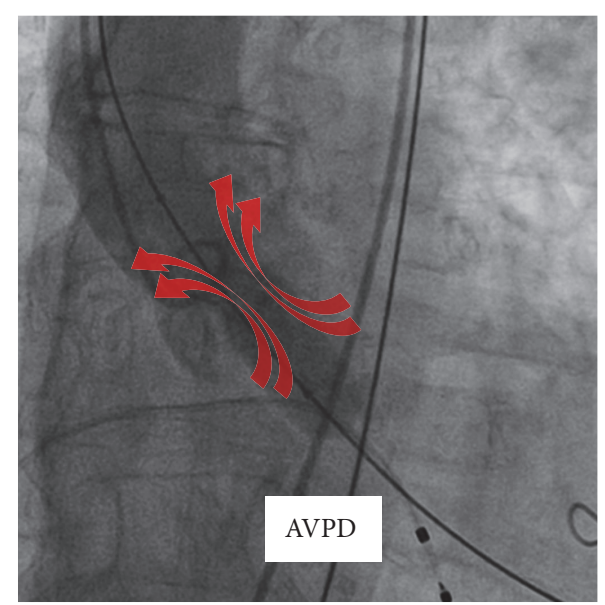

(b)

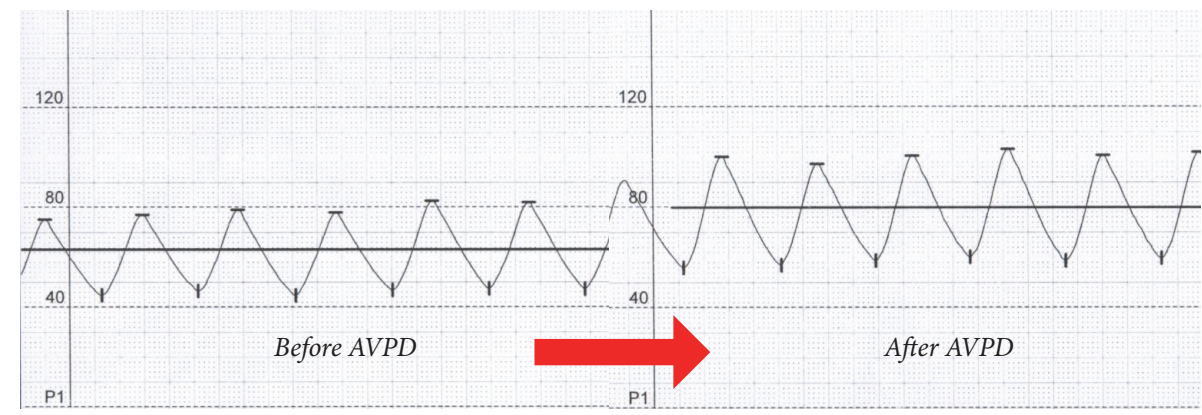

(c)

FIGURE 2: (a) BAV with a normal "occlusive" valvuloplasty-balloon. (b) AVPD with a small, "nonocclusive"-balloon. (c) Hemodynamic effects of AVPD.

or peak velocity $<3 \mathrm{~m} / \mathrm{s}$ and no moderate or severe prosthetic valve regurgitation) [6]. Procedural mortality was defined as all-cause mortality within 30 days or during the index procedure hospitalization [6].

2.4. Statistical Analysis. Continuous data are presented as mean \pm standard deviation, and categorical data are presented as a number and/or percentages. All analysis was performed using XLSTAT for Microsoft Excel 2010 (Microsoft Corporation, Seattle, WA, USA).

\section{Results}

3.1. Baseline Characteristics. The baseline characteristics of the study population are shown in Table 1 . The mean patient age was $81 \pm 6$. Half our patients were women. The mean logistic EuroSCORE (European System for Cardiac Operative Risk Evaluation) was $13 \pm 9$. The mean AV gradient was $39.9 \pm$ $15.7 \mathrm{mmHg}$ and aortic valve area $0.8 \pm 0.2 \mathrm{~cm}^{2}$, indicating severe aortic stenosis.

3.2. Procedural Data. AVPD was performed using a $12 \times$ $60 \mathrm{~mm}$ or $14 \times 60 \mathrm{~mm}$ balloon in $96 \%$ of the cases, and in 2 cases $(4 \%)$ a $12 \times 40 \mathrm{~mm}$ balloon was used. Procedural data is depicted in Table 2 . The mean contrast volume and fluoroscopy time were $108 \pm 35 \mathrm{~mL}$ and $14 \pm 5$ minutes, respectively; mean procedural duration was $60 \pm 18$ minutes.

3.3. Procedural Outcomes. Device success was achieved in all cases $(100 \%)$. No periprocedural stroke, disabling stroke, or TIA occurred. One case $(2 \%)$ of nondisabling stroke (transient Wernicke aphasia) occurred more than 24 hours after TAVR in a patient with chronic atrial fibrillation. Procedural and 30-day mortality was $0 \%$ (Table 3 ). The patients' hemodynamics was stable during the procedure in all cases without the need for additional inotropic medications during AVPD or THV implantation. The mean AV gradient was reduced from $39.9 \pm 15.7 \mathrm{mmHg}$ to $12.3 \pm 4.7 \mathrm{mmHg}$ after THV implantation; these gradients were measured on pre- and postprocedure echocardiography. No case of aortic regurgitation grade $\geq 2$ occurred (Table 3 ).

\section{Discussion}

TAVR as a treatment choice for severe AS is an excellent option for patients with inoperable or high surgical risk, but periprocedural stroke remains a concern compared to surgical aortic valve replacement [1]. BAV is usually performed to ensure the passage of the THV through the stenotic aortic valve. Another aim of BAV is to improve 
TABLE 1: Demographic and clinical characteristics of the study population.

\begin{tabular}{lc}
\hline \multicolumn{2}{c}{ Baseline characteristics } \\
& $\begin{array}{c}\text { Overall population } \\
(N=50)\end{array}$ \\
\hline Age, yrs & $80.8 \pm 5.9$ \\
Female, $n(\%)$ & $25(50)$ \\
BMI, kg/m2 & $27.3 \pm 5.9$ \\
Diabetes, $n$ (\%) & $18(32)$ \\
Dyslipidemia, $n(\%)$ & $32(64)$ \\
Hypertension, $n(\%)$ & $45(90)$ \\
Tobacco use, $n$ (\%) & $11(22)$ \\
Coronary artery disease, $n(\%)$ & $35(70)$ \\
Prior MI, $n(\%)$ & $3(6)$ \\
Prior CABG, $n(\%)$ & $3(6)$ \\
Prior PCI, $n(\%)$ & $19(38)$ \\
Prior stroke or TIA, $n(\%)$ & $4(8)$ \\
Peripheral vascular disease, $n(\%)$ & $7(14)$ \\
COPD, $n(\%)$ & $7(14)$ \\
Baseline creatinine, mg/dL & $1.1 \pm 0.3$ \\
Logistic EuroSCORE 2 & $5.6 \pm 5.0$ \\
EuroSCORE & $13.1 \pm 8.8$ \\
LVEF, $\%$ & $52.2 \pm 12.5$ \\
Maximum aortic gradient, mmHg & $65.2 \pm 24.0$ \\
Mean aortic gradient, mmHg & $39.9 \pm 15.7$ \\
Aortic valve area, cm ${ }^{2}$ & $0.8 \pm 0.2$ \\
\hline
\end{tabular}

Values are $n(\%)$ or mean \pm standard deviation. BMI = body mass index, $\mathrm{MI}=$ myocardial infarction; $\mathrm{CABG}=$ coronary artery bypass graft, $\mathrm{PCI}$ $=$ percutaneous coronary intervention, TIA $=$ transient ischemic attack, $\mathrm{COPD}=$ chronic obstructive pulmonary disease, and $\mathrm{LVEF}=$ left ventricular ejection fraction.

TABle 2: Procedural data.

\begin{tabular}{lc}
\hline & Procedural data \\
& $\begin{array}{c}\text { Overall population } \\
(N=50)\end{array}$ \\
\hline Contrast volume, $\mathrm{mL}$ & $107.9 \pm 35.3$ \\
Radiation dose, Gy & $32402.5 \pm 26989.7$ \\
X-ray time, min & $13.9 \pm 5.2$ \\
Length of procedure, min & $59.9 \pm 17.8$ \\
Balloon Size, $n(\%)$ & \\
$12 \times 40 \mathrm{~mm}$ & $2(4)$ \\
$12 \times 60 \mathrm{~mm}$ & $32(64)$ \\
$14 \times 60 \mathrm{~mm}$ & $16(32)$ \\
Valve Size, $n(\%)$ & \\
SAPIEN $323 \mathrm{~mm}$ & $22(44)$ \\
SAPIEN $326 \mathrm{~mm}$ & $19(38)$ \\
SAPIEN $329 \mathrm{~mm}$ & $9(18)$ \\
\hline
\end{tabular}

Values are $n(\%)$ or mean \pm SD. F $=$ French.
TABLE 3: Procedural outcomes.

\begin{tabular}{lc}
\hline \multicolumn{2}{c}{ Procedural outcomes } \\
Complication, $n(\%)$ & $\begin{array}{c}\text { Overall population } \\
(N=50)\end{array}$ \\
\hline Device success & $50(100)$ \\
Aortic regurgitation $\geq$ grade 2 & $0(0)$ \\
Mean AV gradient, mmHg & $12.3 \pm 4.7$ \\
Myocardial infarction & $1(2)$ \\
Periprocedural stroke & $0(0)$ \\
Disabling stroke & $0(0)$ \\
Nondisabling stroke & $1(2)$ \\
TIA & $0(0)$ \\
BARC life threatening/disabling bleeding & $0(0)$ \\
BARC major bleeding & $4(8)$ \\
BARC minor bleeding & $5(10)$ \\
VARC2 major vascular complication & $0(0)$ \\
VARC2 minor vascular complication & $7(14)$ \\
Permanent pacemaker implant & $8(16)$ \\
Acute kidney injury (RIFLE & $5(10)$ \\
Classification) & $2(4)$ \\
Stage 1 (risk) & $3(6)$ \\
Stage 2 (injury) & $0(0)$ \\
Stage 3 (failure) & $0(0)$ \\
Procedural mortality &
\end{tabular}

Values are $n(\%) . \mathrm{AV}=$ aortic valve, TIA = transient ischemic attack, BARC $=$ bleeding academic research consortium, VARC $=$ valve academic research consortium, RIFLE = risk, injury, failure, loss of kidney function, and endstage kidney disease.

the compliance of the rigid, calcified $\mathrm{AV}$ leaflets and to increase the aortic valve area $[7,8]$. With BAV, a sufficient prosthesis deployment is assured, which is of particular importance for self-expanding THV exerting less radial force. Finally, BAV may be used as an additional sizing modality for prosthesis selection in cases with borderline or questionable annulus size. The mechanisms underlying successful BAV are mainly commissural separation and perhaps more importantly intraleaflet fracturing of calcific nodules [8-10]. Microembolization of debris is thought to occur during multiple phases of the procedure, such as crossing the aortic valve and ascending aorta with different wires, performing $\mathrm{BAV}$, and positioning of the THV $[4,11]$. These microembolizations may account to strokes associated with TAVR. Multiple studies have identified silent diffuse embolic strokes on magnetic resonance imaging studies [5]. Moreover, prior studies have demonstrated the advantages of avoiding BAV in reducing stroke $[12,13]$. Reports have also been published on deleterious outcomes with BAV prior to TAVR [14]. A current meta-analysis suggested a 30-day stroke rate between $3.2 \%$ and $4.2 \%$ using the Edwards Sapien valve [15]. A recently published multicenter trial using the Edwards Sapien 3 valve observed a 30 -day mortality of $2.1 \%$ and a rate of disabling strokes of $0 \%$ [16]. The nondisabling stroke rate was $1 \%$ in their transfemoral cohort [16]. 
One drawback of BAV is the need for rapid ventricular pacing to allow hemodynamic arrest of the heart. This ensures optimal balloon positioning and avoids excessive movements of the balloon during inflation. These balloon movements may also contribute to embolization of calcified material from the stenotic valve [8]. However, rapid left ventricular pacing carries several risks. First, it may induce ventricular fibrillation in rare cases [17]. Second, it purposely decreases cardiac output temporarily with the potential of hemodynamic instability, especially in patients with aortic stenosis and hypertrophied left ventricle [18]. Previous studies have also shown that rapid pacing is associated with smaller postBAV AV area compared to BAV alone [7, 13]. One explanation of this phenomenon may be that rapid ventricular pacing provokes temporary myocardial stunning, thereby decreasing contractility and flow, resulting in the calculation of a smaller aortic valve area [18]. In patients with severely reduced LV function, any additional rapid pacing may be harmful due to this myocardial stunning. These patients might benefit from performing AVPD without rapid ventricular pacing instead of traditional BAV.

In recent reports, operators perform direct TAVR without $\mathrm{BAV}$, also avoiding rapid ventricular pacing [13]. No increase in adverse outcomes was seen with direct valve implantation of the Edwards Sapien XT valve [13]. It should be noted, however, that these were patients with low to moderate calcification of the AV. Furthermore, it has to be considered that most of the TAVR valves cannot be withdrawn once they have entered the body. Thus, failure of crossing the aortic valve without predilatation makes the procedure much more complex. Our prospective analysis shows excellent procedural success and device success rates using AVPD without rapid pacing (Table 2). Passage of the THV through the stenotic AV was achieved on the first attempt in all patients, even in cases with severely calcified valves. In each case, single balloon dilation was employed and the rate of clinical stroke was very low. During the 30 day period, we observed no disabling stroke, and only one nondisabling stroke that happened more than $24 \mathrm{~h}$ after TAVR in a patient with chronic atrial fibrillation. Despite the small study population, this allows us to hypothesize that performing AVPD with a small balloon is less aggressive in comparison to BAV and may reduce mortality as well as both the degree of microembolization and clinical stroke. However, larger cohorts and corroborative imaging will be needed for a more definitive conclusion about the impact of AVPD on stroke and mortality.

\section{Study Limitations}

This prospective study reflects a single-center experience using AVPD in a limited number of patients without a control group. Since this is not a randomized study, selection bias is inherent and may have influenced our findings.

\section{Conclusion}

The use of small balloons for AVPD prior to positioning and implantation of a transfemoral THV is a safe and feasible technique. It eliminates the need for rapid pacing without compromising procedural outcomes. Improvement in the TAVR procedure with refinement of the device is promising, but the advancements in procedural techniques described here are also necessary to improve patient outcomes. Future randomized trials are required to determine the impact of AVPD without rapid pacing in comparison to the traditional BAV and direct THV implantation on hemodynamic and clinical outcomes, especially on silent and clinical cerebral ischemia.

\section{Conflicts of Interest}

Dr. Albert Markus Kasel is a medical consultant for and receives research support from Edwards Life Sciences. The other authors have no conflicts of interest to disclose.

\section{Acknowledgments}

The authors would like to express their gratitude to the staff of the intensive care unit/cardiac catheterization laboratory and to their cardiac surgery and anesthesia colleagues.

\section{References}

[1] M. B. Leon, C. R. Smith, and M. Mack, "Transcatheter aorticvalve implantation for aortic stenosis in patients who cannot undergo surgery," The New England Journal of Medicine, vol. 363, no. 17, pp. 1597-1607, 2010.

[2] A. Cribier, "Development of transcatheter aortic valve implantation (TAVI): a 20-year odyssey," Archives of Cardiovascular Diseases, vol. 105, no. 3, pp. 146-152, 2012.

[3] A. Cribier, P.-Y. Litzler, H. Eltchaninoff et al., "Technique of transcatheter aortic valve implantation with the edwardssapien ${ }^{\circledR}$ heart valve using the transfemoral approach," Herz, vol. 34, no. 5, pp. 347-356, 2009.

[4] P. Kahlert, F. Al-Rashid, P. Döttger et al., "Cerebral embolization during transcatheter aortic valve implantation: a transcranial doppler study," Circulation, vol. 126, no. 10, pp. 1245-1255, 2012.

[5] P. Kahlert, S. C. Knipp, M. Schlamann et al., "Silent and apparent cerebral ischemia after percutaneous transfemoral aortic valve implantation: a diffusion-weighted magnetic resonance imaging study," Circulation, vol. 121, no. 7, pp. 870-878, 2010.

[6] A. P. Kappetein, S. J. Head, P. Généreux et al., "Updated standardized endpoint definitions for transcatheter aortic valve implantation: the valve academic research consortium-2 consensus document," Journal of the American College of Cardiology, vol. 60, no. 15, pp. 1438-1454, 2012.

[7] I. Ben-Dor, A. D. Pichard, L. F. Satler et al., "Complications and outcome of balloon aortic valvuloplasty in high-risk or inoperable patients," JACC: Cardiovascular Interventions, vol. 3, no. 11, pp. 1150-1156, 2010.

[8] H. Hara, W. R. Pedersen, E. Ladich et al., "Percutaneous balloon aortic valvuloplasty revisited: time for a renaissance?" Circulation, vol. 115, no. 12, pp. e334-e338, 2007.

[9] J. M. Isner, D. A. Samuels, G. A. Slovenkai et al., "Mechanism of aortic balloon valvuloplasty: fracture of valvular calcific deposits," Annals of Internal Medicine, vol. 108, no. 3, pp. 377380, 1988. 
[10] R. G. McKay, R. D. Safian, A. J. E. Lock et al., "Balloon dilatation of calcific aortic stenosis in elderly patients: postmortem, intraoperative, and percutaneous valvuloplasty studies," Circulation, vol. 74, no. 1, pp. 119-125, 1986.

[11] T. Drews, M. Pasic, S. Buz et al., "Transcranial Doppler sound detection of cerebral microembolism during transapical aortic valve implantation.", The Thoracic and Cardiovascular Surgeon, vol. 59, no. 4, pp. 237-242, 2011.

[12] W. R. Davies, V. N. Bapat, J. E. Hancock, C. P. Young, S. R. Redwood, and M. R. Thomas, "Direct TAVI using a balloon-expandable system: a novel technique to eliminate predeployment balloon aortic valvuloplasty," EuroIntervention, vol. 10, no. 2, pp. 248-252, 2014.

[13] E. Garcia, P. Martin, R. Hernandez et al., "Feasibility and safety of transfemoral implantation of edwards sapien xt prosthesis without balloon valvuloplasty in severe stenosis of native aortic valve," Catheterization and Cardiovascular Interventions : Official Journal of the Society for Cardiac Angiography Interventions, vol. 83, pp. 791-795, 2014.

[14] K. D. Algarni, K. L. Greason, R. M. Suri, H. I. Michelena, and J. F. Maalouf, "Aborted TAVR following aortic balloon valvuloplasty," Journal of Cardiac Surgery, vol. 30, no. 3, pp. 251252, 2015.

[15] G. Athappan, R. D. Gajulapalli, P. Sengodan et al., "Influence of transcatheter aortic valve replacement strategy and valve design on stroke after transcatheter aortic valve replacement: a meta-analysis and systematic review of literature," Journal of the American College of Cardiology, vol. 63, no. 20, pp. 2101-2110, 2014.

[16] J. Webb, G. Gerosa, T. Lefèvre et al., "Multicenter evaluation of a next-generation balloon-expandable transcatheter aortic valve," Journal of the American College of Cardiology, vol. 64, no. 21, pp. 2235-2243, 2014.

[17] J. N. Weiss, Z. Qu, P. S. Chen et al., “The dynamics of cardiac fibrillation," Circulation, vol. 112, no. 8, pp. 1232-1240, 2005.

[18] C. Witzke, C. W. Don, R. J. Cubeddu et al., "Impact of rapid ventricular pacing during percutaneous balloon aortic valvuloplasty in patients with critical aortic stenosis: Should we be using it?" Catheterization and Cardiovascular Interventions, vol. 75 , no. 3, pp. 444-452, 2010. 


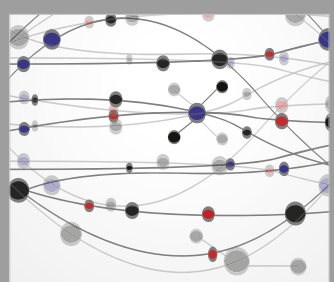

The Scientific World Journal
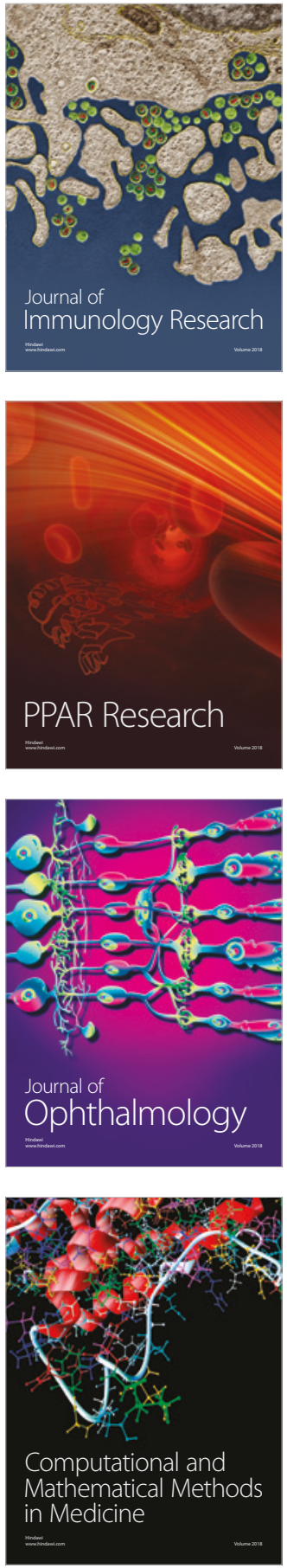

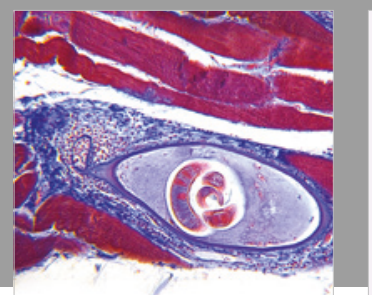

Gastroenterology Research and Practice

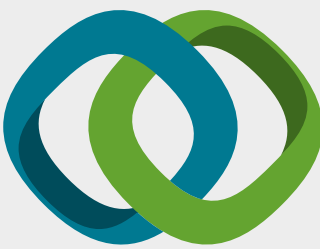

\section{Hindawi}

Submit your manuscripts at

www.hindawi.com
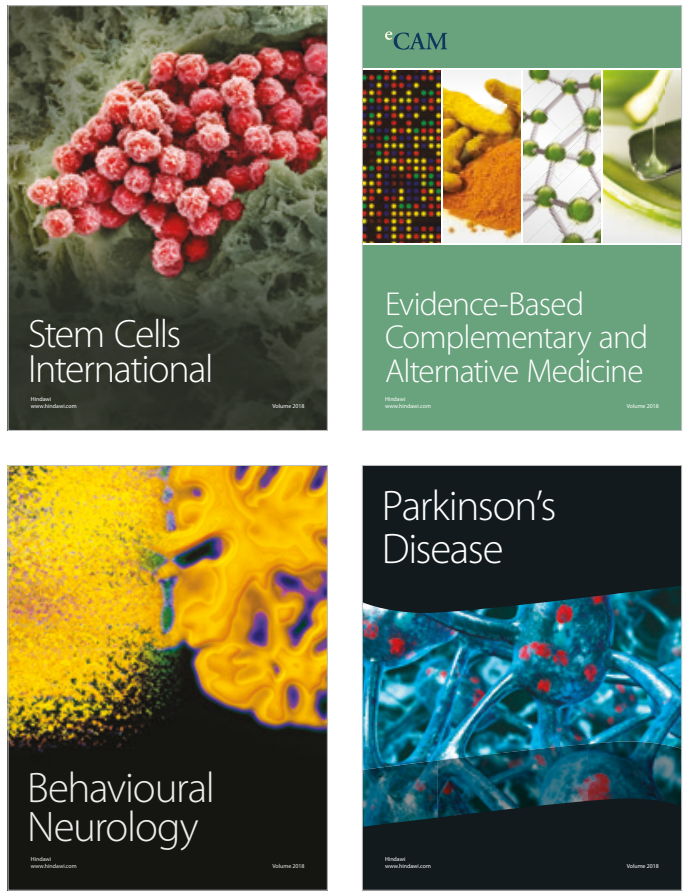

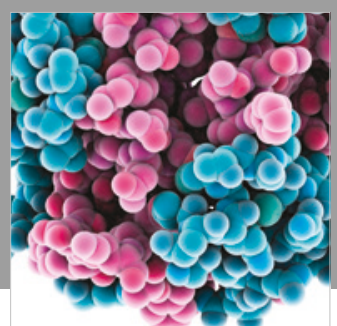

ournal of

Diabetes Research

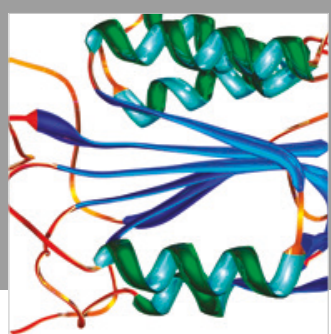

Disease Markers
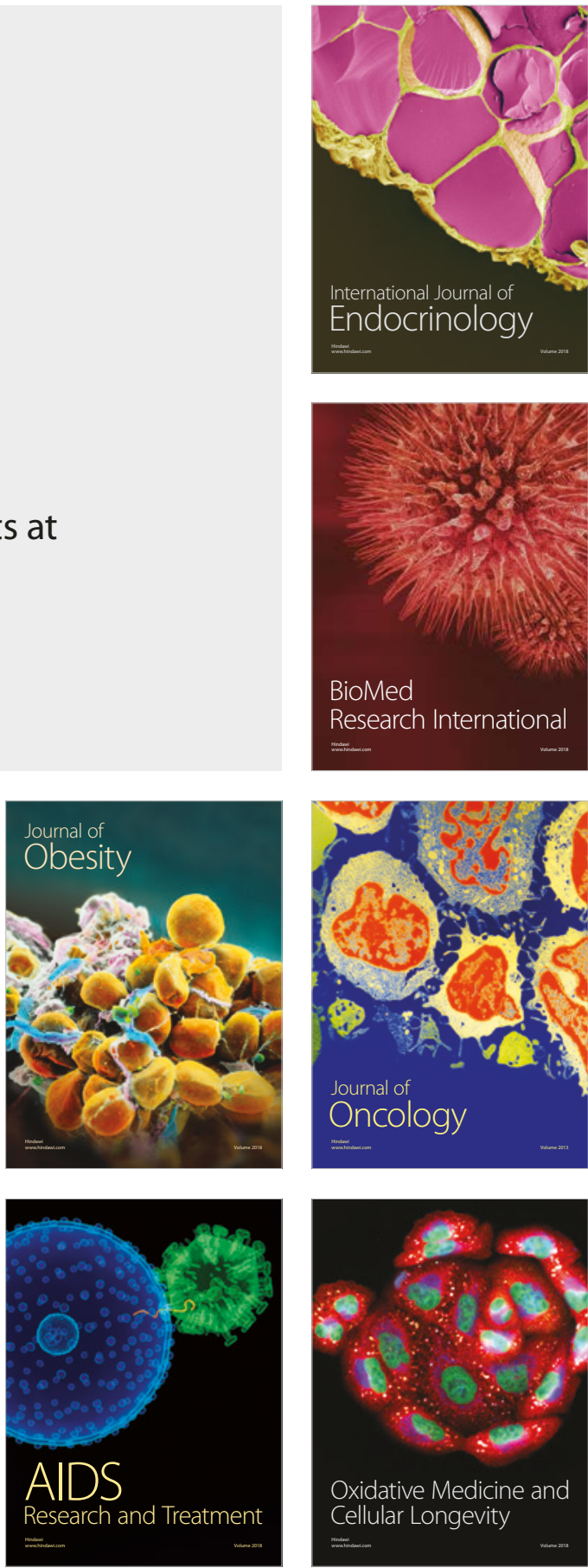